Old Dominion University

ODU Digital Commons

Physics Faculty Publications

Physics

2001

\title{
Coherent-Path Model for Nuclear Resonant Scattering of Gamma Radiation From Nuclei Excited by Synchrotron Radiation
}

Gilbert R. Hoy

Old Dominion University

Jos Odeurs

Romain Coussement

Follow this and additional works at: https://digitalcommons.odu.edu/physics_fac_pubs

Part of the Nuclear Commons, and the Quantum Physics Commons

\section{Repository Citation}

Hoy, Gilbert R.; Odeurs, Jos; and Coussement, Romain, "Coherent-Path Model for Nuclear Resonant Scattering of Gamma Radiation From Nuclei Excited by Synchrotron Radiation" (2001). Physics Faculty Publications. 210.

https://digitalcommons.odu.edu/physics_fac_pubs/210

\section{Original Publication Citation}

Hoy, G. R., Odeurs, J., \& Coussement, R. (2001). Coherent-path model for nuclear resonant scattering of gamma radiation from nuclei excited by synchrotron radiation. Physical Review B, 63(18), 184435. doi:10.1103/PhysRevB.63.184435

This Article is brought to you for free and open access by the Physics at ODU Digital Commons. It has been accepted for inclusion in Physics Faculty Publications by an authorized administrator of ODU Digital Commons. For more information, please contact digitalcommons@odu.edu. 


\title{
Coherent-path model for nuclear resonant scattering of gamma radiation from nuclei excited by synchrotron radiation
}

\author{
Gilbert R. Hoy, ${ }^{1}$ Jos Odeurs,${ }^{2}$ and Romain Coussement ${ }^{2}$ \\ ${ }^{1}$ Physics Department, Old Dominion University, Norfolk, Virginia 23529-0116 \\ ${ }^{2}$ Katholieke Universiteit Leuven, Instituut voor Kern-en Stralingsfysica, Celestijnenlaan 200 D, \\ B-3001 Leuven, Belgium
}

(Received 23 October 1996; published 24 April 2001)

\begin{abstract}
Previous theoretical descriptions of nuclear resonant scattering of synchrotron radiation have been based on the semiclassical optical model or on several quantum mechanical models. These models are fine but do not give a clear physical picture of all the processes. The theory presented here gives a clear physical picture of all the relevant aspects of nuclear resonant scattering. The model treats the nuclear resonant sample as a onedimensional chain of "effective" nuclei. However, the model is deceptive. It only appears to be one dimensional. It actually treats the sample as a series of "effective" planes. The analysis uses the time-dependent quantum mechanical techniques due to Heitler. A closed form solution, for the time-dependent forwardscattered intensity, is found. The only parameter in the theory is $N$ the number of "effective" nuclei (planes) in the model. It is shown that the prominent experimental features, the "speed-up" and "dynamical beat" effects, are primarily due to a $\pi$ phase change of reemitted radiation, compared to the incident radiation, that occurs when radiation is absorbed and reemitted without recoil by a single "effective" nucleus (plane). The model also predicts results for the incoherent processes.
\end{abstract}

DOI: $10.1103 /$ PhysRevB.63.184435

PACS number(s): 76.80. $+\mathrm{y}, 78.90 .+\mathrm{t}, 42.25 . \mathrm{Bs}$

\section{INTRODUCTION}

Since 1985, when the first unambiguous observation of nuclear-resonant excitation of nuclei using synchrotron radiation ${ }^{1}$ was achieved, there has been significant progress made in this field. A review ${ }^{2}$ of this subject area contains a summary of early experimental results, as well as many references to the original important papers in the field.

Theoretical studies of such nuclear-resonant scattering have resulted in a series of papers. A classical optical model, ${ }^{2}$ as well as more fundamental models, has been developed. The fundamental approach presents the idea of a delocalized excitation spread out over all resonant nuclei, the so-called nuclear "exciton.' This idea was introduced by Hannon and Trammell ${ }^{3-7}$ and by Kagan and Afanas'ev. ${ }^{8-11}$ Hannon and Trammell developed the dynamical theory of gamma-ray optics utilizing multiple scattering equations and Gerdau $^{12}$ followed the approach by Hannon and Trammell in their development of a computer program (CONUSS) which permits the evaluation of time-differential spectra of nuclearresonant scattering of synchrotron radiation. The program package can be used to interpret either Bragg or forward scattering. In the work of Kagan and Afanas'ev the radiation field in the crystal is described by the usual set of (classical) Maxwell equations (an extension of the Laue theory of x-ray scattering ${ }^{13}$ ). Solutions for the radiated intensity as a function of time are obtained for scattering in the forward or in Bragg directions.

In this paper nuclear-resonant scattering of synchrotron radiation will be treated according to time-dependent quantum mechanics in the frequency domain using Fourier transformations. The first step in the analysis is to obtain the set of coupled equations relating all possible amplitudes. This will be done in three dimensions first. These equations are equivalent to the familiar multiple scattering equations. ${ }^{3-7}$ Arguments will be given to show why the analysis is performed assuming forward scattering. The problem of multiple scattering does not, in general, give an easily interpretable analytic solution for the radiated intensity. It turns out that an analytical expression for the forward-radiated intensity can be found with our approach. This analytic solution is found because a recursion relation exists between the amplitudes, in the frequency domain, for the absorption and emission of radiation. This leads to an exact calculation of all amplitudes. The Fourier transform of the amplitude for emission of radiation gives the amplitude, in the time domain, for each frequency component. This permits the construction of the plane-wave packet, representing the forward-scattered radiation, by weighting each plane-wave frequency component according to its calculated amplitude. From this result the intensity of radiation reaching a detector can be calculated analytically. The solution of the problem is exact, in the context of the model, and is expressed as a finite series. Each term in the series corresponds to particular "path" the radiation takes in reaching the detector. Each path corresponds to a definite number of multiple forward-scattering events. The number of terms in the series, i.e., the number of paths, depends on $N$, the sample "thickness" in the model. The new solution is quite different in character from all previous solutions, although it agrees with them quantitatively. Our approach leads to a physical understanding of how the various multiple scattering processes relate to the observed forwardradiated intensity.

Our model can also treat incoherent processes. In any multiple scattering path one must consider the cases where a sequence of scattering events occurs without recoil, i.e., coherent events, but then terminates in a final scattering process that is incoherent. This last event in the path produces 
the detected "particle" which can be a gamma ray, emitted with recoil, or a conversion electron. Therefore the coherence of the first steps in the path will influence the observation of the incoherent intensity. Such paths can be easily treated using the methods developed here.

The details of the calculation are contained in Appendixes $\mathrm{A}$ and $\mathrm{B}$. The principal result for the recoil-free case is given in Eq. (27). Quantum-beat effects are contained in Eqs. (30) and (31). Incoherent processes are described by Eqs. (32) and (33). Two examples of incoherent processes are treated. Processes with recoil are described by Eqs. (34) and (35) and those involving the conversion-electron channel are also described by Eqs. (34) and (35) after a slight modification.

\section{MATHEMATICAL BACKGROUND}

The general method used in this paper is discussed in Heitler, ${ }^{14}$ Harris, ${ }^{15}$ and Hoy. ${ }^{16}$ Reference 16 treats the radioactive-source case. The method applies time-dependent quantum mechanics in the frequency domain to obtain a set of coupled equations. The Hamiltonian of the system is divided into two parts. $H_{0}$ is the part that describes the evolution of the nuclear states and the free radiation field in the absence of coupling between the nuclear states and the radiation field. The eigenstates of $H_{0}$ correspond in this case to nuclear states of an ensemble of nuclei, and the states of the free radiation field are taken here as plane waves. Any excited nuclear state can be located at any one of the nuclear positions in the medium. $V$ denotes the part of the Hamiltonian responsible for inducing transitions between the nuclear levels.

The actual state of the system is then expressed as

$$
|\Psi(t)\rangle=\sum_{l} a_{l}(t) e^{-i\left(E_{l} t / \hbar\right)}\left|\varphi_{l}(0)\right\rangle,
$$

where $\left|\varphi_{l}(0)\right\rangle$ is an eigenstate of $H_{0}$. Solving the Schrödinger equation leads to a set of coupled differential equations relating the expansion coefficients $a_{l}(t)$.

$$
i \hbar \frac{d a_{l}}{d t}=\sum_{q} a_{q}(t) e^{i\left(\omega_{l}-\omega_{q}\right) t}\left\langle\varphi_{l}(0)|V| \varphi_{q}(0)\right\rangle+i \hbar \delta_{\ln } \delta(t)
$$

where $\omega_{l}-\omega_{q}=\left(E_{l}-E_{q}\right) / \hbar$.

The Kronecker delta and the delta function are used to insure that at time $t=0$ the system is the state where $l=n$. Next introducing the Fourier transform

$$
a_{l}(t)=-\frac{1}{2 \pi i} \int_{-\infty}^{\infty} d \omega A_{l}(\omega) e^{i\left(\omega_{l}-\omega\right) t},
$$

Eq. (2) can be rewritten in the frequency domain. The result is

$$
\left(\omega-\omega_{l}+i \varepsilon\right) A_{l}(\omega)=\sum_{q} A_{q}(\omega) \frac{V_{l q}}{\hbar}+\delta_{\ln },
$$

where $V_{l q}$ is the matrix element inducing a transition from the $q$ th state of $H_{0}$ to the $l$ th state of $H_{0}$, and a pole is introduced into the lower half of the complex plane $(\varepsilon>0)$ to insure that all amplitudes $a_{l}(t)$ are zero for $t<0$. The advantage of the set of equations, Eq. (4), is that it is coupled set of linear, not differential, equations. In the next section this general formalism will be applied to the study of the interaction of synchrotron radiation with nuclei embedded in a lattice.

\section{ANALYSIS}

\section{A. Fundamental equations}

As the initial condition we will take all absorber nuclei in the lattice in the ground state and only synchrotron radiation present. This state will be a linear superposition of states such as $\left|G_{0}\right\rangle \otimes\left|0,0, \ldots . .0,1_{\mathbf{k}}, 0, \ldots ..\right\rangle \otimes\left|\left\{0_{\mathbf{k}^{\prime}}\right\}\right\rangle \otimes|[0]\rangle$, where $\left|G_{0}\right\rangle$ represents the state in which all nuclei in the lattice are in the ground state. $\left|\left\{0_{\mathbf{k}^{\prime}}\right\}\right\rangle$ represents the absence of photons other then those of the synchrotron radiation $(\mathrm{SR})$ and $|[0]\rangle$ represents the state with no conversion electrons. $\left|0,0, \ldots . .1_{\mathbf{k}}, 0, \ldots ..\right\rangle$ is the state of a $\mathrm{SR}$ photon having wave vector $\mathbf{k}$. The polarization of the SR photons has been omitted. It will be shown later how the polarization can be included. The state vector of the complete system can be written as a linear combination of all possible states. To keep the analysis as simple as possible, absorption and emissions with recoil will be omitted temporarily. It will become clear, later, how processes with recoil can be incorporated into the model. Besides the initial state given above, the complete system can be found in a variety of other states, which will be defined below.

The nucleus labeled $m$ at position $\mathbf{r}_{m}$ can be excited (with some probability amplitude) to one of its excited states $\left|e_{m}^{j}\right\rangle$ with energy $\hbar \omega_{j}$, due to the SR pulse, while all other nuclei remain in the ground state and no photons or conversion electrons are present. This state will be denoted $\left|e_{m}^{j}\right\rangle \otimes|G(m)\rangle \otimes\left|\left\{0_{\mathbf{k}}\right\}\right\rangle \otimes\left|\left\{0_{\mathbf{k}^{\prime}}\right\}\right\rangle \otimes|[0]\rangle \quad$ where $\left|\left\{0_{\mathbf{k}}\right\}\right\rangle$ stands for the absence of the SR photon k. Another possible state is the one where all nuclei are in the ground state with no conversion electrons present and only a non-SR-photon present. This state will be denoted $\left|G_{0}\right\rangle$ $\otimes\left|\left\{0_{\mathbf{k}}\right\}\right\rangle \otimes\left|0,0, \ldots ., 0,1_{\mathbf{k}^{\prime}}, 0, \ldots ..\right\rangle \otimes|[0]\rangle$. The ensemble of these states forms a continuum because all possible k's must, in general, be considered. Finally one has the state with all nuclei in the ground state, no photons and only a conversion electron is present. This state is denoted $\left|G_{0}\right\rangle \otimes\left|\left\{0_{\mathbf{k}}\right\}\right\rangle$ $\otimes\left|\left\{0_{\mathbf{k}^{\prime}}\right\}\right\rangle \otimes\left|1_{\mathbf{p}, m}\right\rangle .\left|1_{\mathbf{p}, m}\right\rangle$ denotes the state of the conversion electron (from nucleus $m$ ) having momentum $\mathbf{p}$ and energy $\hbar \omega_{\mathbf{p}}$. The polarization of the conversion electron has been omitted.

The general state vector of the system is then 


$$
\begin{aligned}
& |\psi(t)\rangle=\sum_{\mathbf{k}}^{\prime} a_{\mathbf{k}}(t) e^{-i \omega_{\mathbf{k}^{t}}}\left|G_{0}\right\rangle \otimes\left|0,0, \ldots ., 0,1_{\mathbf{k}}, 0, \ldots . .\right\rangle \otimes\left|\left\{0_{\mathbf{k}}\right\}\right\rangle \otimes|[0]\rangle+\sum_{m=1}^{N} \sum_{j} b_{m, j}(t) e^{-i \omega_{j} t}\left|e_{m}^{j}\right\rangle \otimes|G(m)\rangle \otimes\left|\left\{0_{\mathbf{k}}\right\}\right\rangle \otimes\left|\left\{0_{\mathbf{k}^{\prime}}\right\}\right\rangle \\
& \otimes|[0]\rangle+\sum_{\mathbf{k}^{\prime}} c_{\mathbf{k}^{\prime}}(t) e^{-i \omega_{\mathbf{k}^{\prime}} \mid}\left|G_{0}\right\rangle \otimes\left|\left\{0_{\mathbf{k}}\right\}\right\rangle \otimes\left|0,0, \ldots . .0,1_{\mathbf{k}^{\prime}}, 0, \ldots . .\right\rangle \otimes|[0]\rangle+\sum_{\mathbf{p}} \sum_{m=1}^{N} d_{\mathbf{p}, m}(t) e^{-i \omega_{\mathbf{p}} t}\left|G_{0}\right\rangle \otimes\left|\left\{0_{\mathbf{k}}\right\}\right\rangle \\
& \otimes\left|\left\{0_{\mathbf{k}^{\prime}}\right\}\right\rangle \otimes\left|1_{\mathbf{p}, m}\right\rangle .
\end{aligned}
$$

The second series in Eq. (5) is the usual so-called exciton state mentioned above. The prime on the first summation symbol of Eq. (5) means that the sum on $\mathbf{k}$ is restricted by the properties of the synchrotron-radiation source. The coefficients $\left\{a_{\mathbf{k}}(t), b_{m, j}(t), c_{\mathbf{k}^{\prime}}(t), d_{\mathbf{p}, m}(t)\right\}$ have to be determined by solving the Schrödinger equation using time-dependent quantum mechanics. This will give a system of coupled differential equations, i.e., Eq. (2) specialized to this particular problem. The Hamiltonian of the system is the sum of $H_{0}$, describing the nuclei in the lattice and the radiation field without any coupling, and $V$ which causes the transitions between nuclear levels. Thus $V$ takes account of the absorption of the SR photons by the nuclei as well as the emission and absorption by the nuclei of non-SR-photons, i.e., multiple scattering processes. According to standard expressions for emission and absorption ${ }^{14}$ the matrix element describing absorption of a photon with wave vector $\mathbf{k}$ by a nucleus at $\mathbf{r}_{m}$ contains the phase factor $e^{i \mathbf{k} \cdot \mathbf{r}_{m}}$, while the remaining factor will be denoted $V_{\mathbf{k}, j}$. For emission at $\mathbf{r}_{m}$ one has the factors $e^{-i \mathbf{k} \cdot \mathbf{r}_{m}}$ and $V_{\mathbf{k}, j}^{*}$.

As already mentioned, the introduction of the Fourier transform of these coefficients yields a system of linear coupled equations. In the Fourier domain the amplitudes will now be a function of $\omega$ [see Eq. (4)]. At time $t=0$ the synchrotron radiation is present and all absorber nuclei are in the ground state. We assign this condition an amplitude $A_{\mathbf{k}}(\omega)$ $=A$, i.e., a constant, having the dimensions of seconds. The frequency dependence of the synchrotron radiation is taken as constant because the synchrotron radiation pulse is essentially a delta function in time for the cases we treat here. (A digression on this point will be made at the end of Appendix B.) The amplitude corresponding to excitation of the $m$ th absorber nucleus located at $\mathbf{r}_{m}$ to one of its excited states $\hbar \omega_{j}$ and no photons or conversion electrons present is $B_{m, j}(\omega)$. At this stage of the analysis just one excited state is considered. This means that quantum beats are not yet considered. It will become clear, later, how quantum beats can be included in the model. Also we let $C_{\mathbf{k}^{\prime}}(\omega)$ correspond to the amplitude when all absorber nuclei are in the ground state, there are no conversion electrons, and a non-SR-pulse photon is present. Finally we define the amplitude $D_{m, \mathbf{p}}(\omega)$ as corresponding to the presence of a conversion electron from the $m$ th nucleus, all absorber nuclei in their ground state and no photons are present. The coupled equations relating these amplitudes can then be shown to be

$$
A_{\mathbf{k}}(\omega)=A
$$

$$
\begin{aligned}
\left(\omega-\omega_{j}+i \varepsilon\right) B_{m, j}(\omega)= & A \sum_{\mathbf{k}} \frac{V_{\mathbf{k}, j}}{\hbar} e^{i \mathbf{k} \cdot \mathbf{r}_{m}} \\
& +\sum_{\mathbf{k}^{\prime}} C_{\mathbf{k}^{\prime}}(\omega) \frac{V_{\mathbf{k}^{\prime}, j}}{\hbar} e^{i \mathbf{k}^{\prime} \cdot \mathbf{r}_{m}} \\
& +\sum_{\mathbf{p}} D_{m, \mathbf{p}} \frac{V_{\mathbf{p}}}{\hbar} e^{i\left(\mathbf{p} \cdot \mathbf{r}_{m} / \hbar\right)}, \\
\left(\omega-\omega_{\mathbf{k}^{\prime}}+i \varepsilon\right) C_{\mathbf{k}^{\prime}}(\omega)= & \sum_{m} B_{m, j}(\omega) \frac{V_{\mathbf{k}^{\prime}, j}^{*}}{\hbar} e^{-i \mathbf{k}^{\prime} \cdot \mathbf{r}_{m}}, \\
\left(\omega-\omega_{\mathbf{p}}+i \varepsilon\right) D_{m, \mathbf{p}}(\omega)= & B_{m, j}(\omega) \frac{V_{\mathbf{p}}^{*}}{\hbar} e^{-i\left(\mathbf{p} \cdot \mathbf{r}_{m} / \hbar\right)},
\end{aligned}
$$

where, as already mentioned, $V_{\mathbf{k}, j}$ corresponds to absorption and $V_{\mathbf{k}, j}^{*}$ corresponds to emission of a photon in the $j$ th transition, and a similar notation is used for the conversion electron. Equation (9) represents the emission of a conversion electron by the nucleus located at $\mathbf{r}_{m}$. In Eq. (7) the prime on the summation symbol representing the sum on $\mathbf{k}$ has been omitted. This will be done for all subsequent summations on k.

An understanding of the structure of these equations can be obtained by considering Eqs. (7) and (8). In Eq. (7) we have the amplitude for finding the $m$ th nucleus in its $j$ th excited state, $B_{m j}$. This can occur several ways. First there can be excitation of that nucleus by the SR pulse which is accounted for by the first term on the right-hand side of Eq. (7). Second, excitation of that nucleus can occur due to radiation coming from deexcitation of one of the other resonant nuclei, the second term on the right-hand side. Finally, excitation of that nucleus can occur by absorption of its own conversion electron, the last term on the right-hand side. Notice the phase factors that must be introduced to specify the location at which each of these processes occurs. Equation (8) expresses the amplitude for finding a non-SR photon present, $C_{\mathbf{k}^{\prime}}$. The term on the right-hand side of Eq. (8) corresponds to having one of the resonant nuclei emit such a photon. Since each nucleus can do this we must sum over all resonant nuclei. Again one must keep track of where that emission took place by introducing the appropriate phase factor.

Solving for $D_{m, \mathbf{p}}(\omega)$ from Eq. (9) and substituting into Eq. (7) gives 


$$
\begin{aligned}
\left(\omega-\omega_{j}+i \varepsilon\right) B_{m, j}(\omega)= & A \sum_{\mathbf{k}} \frac{V_{\mathbf{k}, j}}{\hbar} e^{i \mathbf{k} \cdot \mathbf{r}_{m}} \\
& +\sum_{\mathbf{k}^{\prime}} C_{\mathbf{k}^{\prime}}(\omega) \frac{V_{\mathbf{k}^{\prime}, j}}{\hbar} e^{i \mathbf{k}^{\prime} \cdot \mathbf{r}_{m}} \\
& +B_{m, j} \sum_{\mathbf{p}} \frac{\left|V_{\mathbf{p}}\right|^{2}}{\hbar^{2}\left(\omega-\omega_{\mathbf{p}}+i \varepsilon\right)} .
\end{aligned}
$$

By converting the sum on $\mathbf{p}$ in the last term of the righthand side of Eq. (10) into an integral ${ }^{14}$ and expressing $\left|V_{\mathbf{p}}\right|^{2} / \hbar^{2}\left(\omega-\omega_{\mathbf{p}}+i \varepsilon\right)$ in terms of a principal part and a delta function, one finds it equals

$$
\begin{aligned}
B_{m, j} \sum_{\mathbf{p}} \frac{\left|V_{\mathbf{p}}\right|^{2}}{\hbar^{2}\left(\omega-\omega_{\mathbf{p}}+i \varepsilon\right)} \\
=B_{m, j} \frac{V}{(2 \pi \hbar)^{3}} \frac{1}{\hbar^{2}} P \iiint \frac{\left|V_{\mathbf{p}}\right|^{2}}{\omega-\omega_{\mathbf{p}}} p^{2} d p d \Omega \\
\quad-B_{m, j} i \frac{V}{(2 \pi \hbar)^{3}} \frac{\pi}{\hbar^{2}} \iiint\left|V_{\mathbf{p}}\right|^{2} p^{2} \\
\quad \times \delta\left(\omega-\omega_{\mathbf{p}}\right) d p d \Omega,
\end{aligned}
$$

where $P$ stands for the principal value of the integral. The presence of the volume $V$ inherent in Eq. (11) and in the others, resulting from the conversion of a sum into an integral in three dimensions, is only apparent because the matrix elements such as $\left|V_{\mathbf{p}}\right|^{2}$ contain $^{14} 1 / V$.

When the expression in Eq. (11) is taken to the left-hand side of Eq. (10), the principal value term corresponds to a shift in the frequency that can be incorporated into $\omega_{j}$. The second term of Eq. (11) gives a width due to the interaction of a nucleus with its conversion electron. This width $\gamma_{c}$ is defined by

$$
\gamma_{c}=\frac{2 \pi V}{(2 \pi \hbar)^{3} \hbar} \iiint\left|V_{\mathbf{p}}\right|^{2} p^{2} \delta\left(\omega-\omega_{\mathbf{p}}\right) d p d \Omega .
$$

Rewriting Eq. (10) gives

$$
\begin{aligned}
\left(\omega-\omega_{j}+i \frac{\gamma_{c}}{2 \hbar}\right) B_{m, j}(\omega)= & A \sum_{\mathbf{k}} \frac{V_{\mathbf{k}, j}}{\hbar} e^{i \mathbf{k} \cdot \mathbf{r}_{m}} \\
& +\sum_{\mathbf{k}^{\prime}} C_{\mathbf{k}^{\prime}}(\omega) \frac{V_{\mathbf{k}^{\prime}, j}}{\hbar} e^{i \mathbf{k}^{\prime} \cdot \mathbf{r}_{m}}
\end{aligned}
$$

Solving Eq. (8) for $C_{\mathbf{k}^{\prime}}(\omega)$ and substituting into Eq. (13) gives

$$
\begin{aligned}
\left(\omega-\omega_{j}+i \frac{\gamma_{c}}{2 \hbar}\right) B_{m, j}(\omega) \\
=A \sum_{\mathbf{k}} \frac{V_{\mathbf{k}, j}}{\hbar} e^{i \mathbf{k} \cdot \mathbf{r}_{m}+\sum_{\mathbf{k}^{\prime}} \sum_{m^{\prime}} B_{m^{\prime}, j}(\omega)} \\
\quad \times \frac{\left|V_{\mathbf{k}^{\prime}, j}\right|^{2}}{\hbar^{2}} \frac{1}{\left(\omega-\omega_{\mathbf{k}^{\prime}}+i \varepsilon\right)} e^{i \mathbf{k}^{\prime} \cdot\left(\mathbf{r}_{m}-\mathbf{r}_{m^{\prime}}\right)} .
\end{aligned}
$$

The second series of the right-hand side of Eq. (14) can be divided in two parts: one with $m^{\prime} \neq m$ and the other with $m^{\prime}=m$. Then Eq. (14) can be rewritten as

$$
\begin{aligned}
\left(\omega-\omega_{j}+i \frac{\gamma_{c}}{2 \hbar}\right) B_{m, j}(\omega) \\
=A \sum_{\mathbf{k}} \frac{V_{\mathbf{k}, j}}{\hbar} e^{i \mathbf{k} \cdot \mathbf{r}_{m}+B_{m, j}(\omega)} \\
\quad \times \sum_{\mathbf{k}^{\prime}} \frac{\left|V_{\mathbf{k}^{\prime}, j}\right|^{2}}{\hbar^{2}} \frac{1}{\omega-\omega_{\mathbf{k}^{\prime}}+i \varepsilon}+\sum_{\mathbf{k}^{\prime}} \sum_{m^{\prime} \neq m} B_{m^{\prime}, j}(\omega) \\
\quad \times \frac{\left|V_{\mathbf{k}^{\prime}, j}\right|^{2}}{\hbar^{2}} \frac{1}{\omega-\omega_{\mathbf{k}^{\prime}}+i \varepsilon} e^{i \mathbf{k}^{\prime} \cdot\left(r_{m}-r_{m^{\prime}}\right)} .
\end{aligned}
$$

Considering the second term on the right-hand side of Eq. (15), the sum on $\mathbf{k}^{\prime}$ can be converted into an integral, as done before. This results again in a principal value term and a delta-function term. The principal value term corresponds again to a frequency shift when brought to the left-hand side. The delta function term corresponds to the usual radiative width $^{14} \gamma_{R}$, where

$$
\gamma_{R}=\frac{2 \pi V}{(2 \pi)^{3} \hbar} \iiint\left|V_{\mathbf{k}^{\prime}}\right|^{2} \delta\left(\omega-\omega_{\mathbf{k}^{\prime}}\right) k^{\prime 2} d k^{\prime} d \Omega .
$$

Collecting terms on the left-hand side of Eq. (15) using Eq. (16) gives

$$
\begin{aligned}
\left(\omega-\omega_{j}+i \frac{\Gamma}{2 \hbar}\right) B_{m, j}(\omega) \\
=A \sum_{\mathbf{k}} \frac{V_{\mathbf{k}, j}}{\hbar} e^{i \mathbf{k} \cdot \mathbf{r}_{m}}+\sum_{\mathbf{k}^{\prime}} \sum_{m^{\prime} \neq m} B_{m^{\prime}, j}(\omega) \\
\quad \times \frac{\left|V_{\mathbf{k}^{\prime}, j}\right|^{2}}{\hbar^{2}} \frac{1}{\omega-\omega_{\mathbf{k}^{\prime}}+i \varepsilon} e^{i \mathbf{k}^{\prime} \cdot\left(\mathbf{r}_{m}-\mathbf{r}_{m^{\prime}}\right)},
\end{aligned}
$$

where $\Gamma$ is the total width, equal to the sum of the conversion-electron and radiative widths.

The SR photons have a well-defined direction of propagation. We call this direction the $z$ axis. If one considers scattering in directions other than forward, one must account for the difference in the optical paths from all nuclei in the sample. In perfect crystals one will have at least two "coherent" channels open ${ }^{6}$ if the incident direction of the synchrotron radiation beam relative to the crystal satisfies the Bragg condition. In polycrystalline material emission in cer- 
tain preferential directions, similar to Debye-Scherrer patterns in x-ray diffraction, may exist.

Although nuclear-resonant Bragg scattering of SR from perfect crystals has been observed, the powder pattern peaks from polycrystalline samples have not been observed. Thus it is clear that for polycrystalline material only the forward scattering exhibits significant special features. It has already been established ${ }^{2}$ that specific features, the "speed-up," "dynamical-beat," and "quantum-beats" effects, occur in forward scattering. (These effects will be described below.) In the following we will give an argument that indicates why $C_{\mathbf{k}^{\prime}}(\omega)$ is particularly important for scattering in the forward direction.

Putting Eq. (17) into Eq. (8) gives

$$
\begin{aligned}
C_{\mathbf{k}^{\prime}}(\omega)= & \frac{1}{\left(\omega-\omega_{\mathbf{k}^{\prime}}+i \varepsilon\right)} \frac{1}{\left(\omega-\omega_{j}+i \frac{\Gamma}{2 \hbar}\right)} \\
& \times\left[A \sum_{m} \sum_{\mathbf{k}} \frac{V_{\mathbf{k}, j}}{\hbar} \frac{V_{\mathbf{k}^{\prime}, j}^{*}}{\hbar} e^{i\left(\mathbf{k}-\mathbf{k}^{\prime}\right) \cdot \mathbf{r}_{m}}+\sum_{m} \sum_{m^{\prime} \neq m} B_{m^{\prime}, j}(\omega) \sum_{\mathbf{k}^{\prime \prime}} \frac{\left|V_{\mathbf{k}^{\prime \prime}, j}\right|^{2}}{\hbar^{2}} \frac{e^{i \mathbf{k}^{\prime \prime} \cdot\left(\mathbf{r}_{m}-\mathbf{r}_{m^{\prime}}\right)}}{\omega-\omega_{\mathbf{k}^{\prime \prime}}+i \varepsilon} e^{-i \mathbf{k}^{\prime} \cdot \mathbf{r}_{m}} \frac{V_{\mathbf{k}^{\prime}, j}^{*}}{\hbar}\right] .
\end{aligned}
$$

This is the fundamental expression describing multiple scattering in the frequency domain. The positions of all resonant nuclei appear in this expression. The first double sum describes the interaction of the nuclei with the SR, the second double sum describes the interaction of nuclei with each other through emission and absorption of gamma radiation, i.e., multiple scattering.

The first series on the right-hand side of Eq. (18) contains

$$
S_{1}=\sum_{m} e^{i\left(\mathbf{k}-\mathbf{k}^{\prime}\right) \cdot \mathbf{r}_{m}} .
$$

For a perfect lattice this sum equals the total number of (resonant) nuclei in the lattice when $\mathbf{k}-\mathbf{k}^{\prime}$ is equal to a reciprocal lattice vector or when $\mathbf{k} \approx \mathbf{k}^{\prime}$. When $\mathbf{k}^{\prime}$ is arbitrary $S_{1}$ is zero. ${ }^{17}$ For a polycrystalline material reciprocal lattice vectors cannot be defined. So one expects $S_{1}$ to be negligible in this case unless $\mathbf{k} \approx \mathbf{k}^{\prime}$. This means that one has scattering primarily in the forward direction when using polycrystalline samples. Similarly the second series on the right-hand side of Eq. (18) contains

$$
S_{2}=\sum_{m} e^{i\left(\mathbf{k}^{\prime \prime}-\mathbf{k}^{\prime}\right) \cdot \mathbf{r}_{m}}
$$

Again we suppose that $S_{2}$ is small unless $\mathbf{k}^{\prime} \approx \mathbf{k}^{\prime \prime}$. An iteration technique can be applied to Eq. (18) with the aid of Eqs. (13) and (17), showing that $C_{\mathbf{k}^{\prime}}(\omega)$ is negligible except for the forward direction. In the next section the solution will be given for the scattering in the forward direction.

\section{B. Scattering in the forward direction}

It might be expected that, when considering a real sample, the multiple scattering path mathematical approach might be possible by restricting the scattering to the forward direction. However, Dr. Stan Ruby has, for several years, been working on such a calculation treating the whole three-dimensional lattice. This approach has turned out to be extremely difficult. In our model the lattice is treated as a one-dimensional chain of "effective" nuclei (or planes, see below). We say "effective" nuclei (planes) because the assumption, that a one-dimensional chain (or stack of planes) can represent a three-dimensional solid, is an extreme one. In fact it is not at all obvious that such a model would lead, as it has (see below), to calculated results that agree with experiment and with previous calculated results. The model is deceptive. In fact, instead of thinking of a chain of $N$ effective nuclei, one may think of a stack of $N$ effective planes. The reasons for this are given in the Conclusions section. The agreement with previous theories only becomes apparent when the actual numerical calculations are compared. This is because the form of our final equations is completely different from those derived by others. (A detailed numerical comparison of the coherent-path model with the other theories, for the radioactive source case, is found in Ref. 16.)

As has been mentioned, the direction of the incoming photons is taken as the $z$ axis. Equation (13) specialized for the forward direction gives

$$
\begin{aligned}
\left(\omega-\omega_{j}+i \frac{\gamma_{c}}{2 \hbar}\right) B_{m, j}(\omega)= & A \sum_{k} \frac{V_{k, j}}{\hbar} e^{i k z_{m}} \\
& +\sum_{k^{\prime}} C_{k^{\prime}}(\omega) \frac{V_{k^{\prime}, j}}{\hbar} e^{i k^{\prime} \cdot z_{m}}
\end{aligned}
$$

Equation (8) becomes

$$
C_{k^{\prime}}(\omega)=\frac{1}{\omega-\omega_{k^{\prime}}+i \varepsilon} \sum_{m} B_{m, j}(\omega) \frac{V_{k^{\prime}, j}^{*}}{\hbar} e^{-i k^{\prime} z_{m}}
$$

Substituting from Eq. (22) into Eq. (21) gives 


$$
\begin{aligned}
\left(\omega-\omega_{j}+i \frac{\gamma_{c}}{2 \hbar}\right) B_{m, j}(\omega) \\
=A \sum_{k} \frac{V_{k, j}}{\hbar} e^{i k z_{m}}+\sum_{k^{\prime}} \sum_{m^{\prime}} B_{m^{\prime}, j} \\
\quad \times \frac{\left|V_{k^{\prime}, j}\right|^{2}}{\hbar^{2}} e^{i k^{\prime}\left(z_{m}-z_{m^{\prime}}\right)} \frac{1}{\omega-\omega_{k^{\prime}}+i \varepsilon} .
\end{aligned}
$$

In Appendix $\mathrm{A}$ it is shown how $B_{m, j}(\omega)$ can be found using an iteration procedure. Once $B_{m, j}(\omega)$ is found, $C_{k^{\prime}}(\omega)$ can be calculated from Eq. (22). The Fourier transform of $C_{k^{\prime}}(\omega)$ gives $c_{k^{\prime}}(t)$ from which the photon's wave packet can be constructed. This is also done in Appendix A.

The wave packet describing the forward-scattered photon is given by Eq. (B15) from Appendix B:

$$
\begin{aligned}
\Psi_{j}\left(z, t^{\prime}\right)= & -\frac{L}{c^{2}} \sqrt{\frac{2 \pi c}{\Delta \omega_{p}}} \frac{V_{k_{0, j}} V^{*}\left(\omega_{j}\right)}{\hbar^{2}} e^{-i\left(\omega_{j}-i(\Gamma / 2 \hbar)\right) t^{\prime}} \\
& \times\left[N+\sum_{n=1}^{N-1}\left(\frac{-\gamma_{R}}{2 \hbar}\right)^{n}\left(\begin{array}{c}
N \\
n+1
\end{array}\right) \frac{t^{! n}}{n !}\right] .
\end{aligned}
$$

In Eq. (24), $z$ locates the position of the detector, $t^{\prime}=t$ $-z / c, L$ is the normalizing length which appears when the sums on the discrete variables are transformed into integrals, and $\Delta \omega_{p}$ is the effective bandwidth of the SR pulse. $L$ will disappear from the expressions describing physical processes, as will be shown later. Furthermore, $j$ labels the particular frequency emitted, $N$ is the effective number of resonant nuclei (planes) in the model, $\gamma_{R}$ is the radiative width of the transition, $\Gamma$ is the total width and the factor $N$ over $n$ +1 is the binomial coefficient. We will show in Sec. IV A 1 below how to determine $N$ for an actual sample. With the approximations made in the calculation, the positions of the effective resonant nuclei (planes) do not appear in the solution. In what follows one may think of "effective"' nuclei or "effective" planes interchangeably. For each "path" to the detector one simply needs to keep track of the number of $180^{\circ}$ phase shifts encountered (see below).

Equation (24) will now be investigated in detail. The factors in front of the exponential represent a product of two transition matrix elements; namely, the matrix element for excitation of a nucleus by the synchrotron radiation, $V_{k_{0}, j}$, and the matrix element for emission of radiation by an excited nucleus, $V^{*}\left(\omega_{j}\right)$. The first term inside the brackets corresponds to the "path" where one nucleus absorbs radiation and reemits radiation directly to the detector. Since there are assumed to be an effective number of nuclei (planes), $N$, participating in the medium, this process occurs $N$ times as shown in Eq. (24). The second term in the square brackets, involving the summation, corresponds to the situation in which synchrotron radiation excites one nucleus (plane) and then the subsequently emitted radiation is absorbed and reemitted several times by other nuclei (planes) until a particular nucleus (plane) radiates to the detector. These are multiple scattering processes. To be specific, consider the first term in the summation. This is the $n=1$ term. It corresponds to the path in which the radiation, emitted from the synchrotron-excited nucleus (plane), gets absorbed and reemitted by only one other nucleus (plane) before reaching the detector. For simplicity we say the radiation hopped on the other nucleus (plane). [A hop corresponds to radiation from one nucleus (plane) being absorbed and reemitted by another nucleus (plane).] The weighting of this term, the binomial coefficient, corresponds to the number of ways of selecting one object from $(N-1)$ objects. Notice that each "hop" gives a phase shift of $180^{\circ}$ [the minus sign inside the square brackets of Eq. (24)] with respect to the no-hop processes. Thus one-hop processes have amplitudes that are $180^{\circ}$ out of phase with respect to the amplitude for no-hop processes. The two-hop processes have amplitudes that are in phase with the no-hop-process amplitudes. Each term in the sum corresponds to a particular hopping sequence, or path, the gamma radiation takes in reaching the detector.

The counting rate at a detector corresponding to the wave packet given by Eq. (24) is

$$
I\left(z, t^{\prime}\right)=c\left|\psi_{j}\right|^{2}
$$

Expressing the squares of the absolute value of the matrix elements of Eq. (24) in terms of the radiative width $\gamma_{R}$ [Eq. (A5) of Appendix A], one finds

$$
I\left(z, t^{\prime}\right)=\frac{\pi \gamma_{R}^{2}}{2 \hbar^{2} \Delta \omega_{p}} e^{\Gamma t^{\prime} / \hbar}\left|N+\sum_{n=1}^{N-1}\left(\frac{-\gamma_{R}}{2 \hbar}\right)^{n}\left(\begin{array}{c}
N \\
n+1
\end{array}\right) \frac{t^{\prime n}}{n !}\right|^{2}
$$

Application of Eq. (26) to each physical situation requires that specific modifications be made to the equation. This will be done in the next sections.

\section{DISCUSSION}

\section{A. Coherent processes}

\section{Speedup and dynamical beats}

Consider the case in which the nuclei have only one excited-state level. This means that there is only one resonant frequency and that, for the moment, quantum beats are not considered. If one is considering a recoil-free process, then $\gamma_{R}$ in Eq. (26) must be multiplied by the recoil-free fraction $f$. Again each term in the sum, contained in the square brackets of Eq. (26), corresponds to a particular hopping sequence or "path" the radiation takes to reach the detector. If we consider only the recoil-free processes, i.e., the Mössbauer effect in which radiation is absorbed and reemitted by the nuclei without recoil, it is impossible to determine which path was taken for each count recorded in the detector. Therefore each path of this recoil-free type must be considered as coherent with all other paths. So we must sum over all paths before squaring to obtain the result. The result is then

$$
I\left(z, t^{\prime}\right)=\frac{\pi f^{2} \gamma_{R}^{2}}{2 \hbar^{2} \Delta \omega_{p}} e^{\Gamma t^{\prime} / \hbar}\left|N+\sum_{n=1}^{N-1}\left(\frac{-f \gamma_{R}}{2 \hbar}\right)^{n}\left(\begin{array}{c}
N \\
n+1
\end{array}\right) \frac{t^{\prime n}}{n !}\right|^{2} .
$$


There are several parameters in Eq. (27); the recoil-free fraction $(f)$, the radiative width $\left(\gamma_{R}\right)$, and the effective number of resonant nuclei (planes) in the sample $(N)$. This number $N$ is associated with the thickness of the resonant medium since it is related to the length of the one-dimensional chain of effective nuclei, or alternatively the number of stacked effective planes. $N$ is the only unspecified parameter in the expression for the intensity. When Eq. (27) or similar expressions, such as Eqs. (31), (33), and (35) (see below), are used for fitting data, $N$ is the only free parameter. Two methods can be used to determine the value of $N$. The empirical method, for determining $N$, is to perform an experiment using a sample of a certain thickness. It is then a simple matter to determine the value $N$ that fits the data. The theory scales linearly with thickness, so $N$ can then be determined for any sample thickness of the same material. The theoretical method for determining $N$ for a specific sample is based on the comparison of the results of this coherent-path model with those from the semi-classical optical model. It has been found $^{18}$ that for the coherent forward scattering, the coherent-path model and the semi-classical optical model give identical results even though the equations themselves are quite different. Noting this observation, one can determine the relationship between $N$, in the coherent-path model, and $\beta$ in the semiclassical optical model. To do this we consider the thin absorber limit. In the semiclassical optical model, when the source and absorber are in resonance, this amounts to expanding the $J_{1}$ Bessel function. In the coherent-path model the thin "absorber" limit is necessarily set by putting $N=1$. Comparing the results gives

$$
N=\frac{\beta \Gamma}{2 f \gamma_{R}},
$$

where the actual thickness parameter $\beta$ is equal to $N_{0} f \sigma_{0} d$. Here $N_{0}$ is the number of resonant nuclei $/ \mathrm{cm}^{3}, f$ is the recoilfree fraction, $\sigma_{0}$ is the maximum cross section evaluated on resonance, and $d$ is the thickness of the sample. Due to the nature of the model, $N$ necessarily takes on integer values only. However, the value of the right-hand side of the Eq. (28) is normally not an integer. Thus, one chooses the closest integer value for $N$. It is important to observe that it is only the new solution that allows a clear physical explanation of the most prominent features of the nuclear-resonant forwardscattering process.

Using Eq. (27) model calculations are presented in Fig. 1 for the case of ${ }^{57} \mathrm{Fe}$. The calculations have been normalized to one at time $t^{\prime}=0$. [Actually the value of the forward scattered intensity at $t^{\prime}=0$ is proportional to the thickness squared $\left(N^{2}\right)$.] The results in Fig. 1 show the effect of increasing the thickness of the resonant medium. Notice the experimentally observed "speedup" effect where the intensity radiated in the forward direction decays faster then one would expect according to the lifetime of an isolated nucleus. This effective lifetime (of the exciton in other theories) decreases as the sample thickness increases. In fact, when the sample reaches a certain thickness the decay curve exhibits a local maximum at a time greater than zero. (This effect has also already been observed experimentally.) This

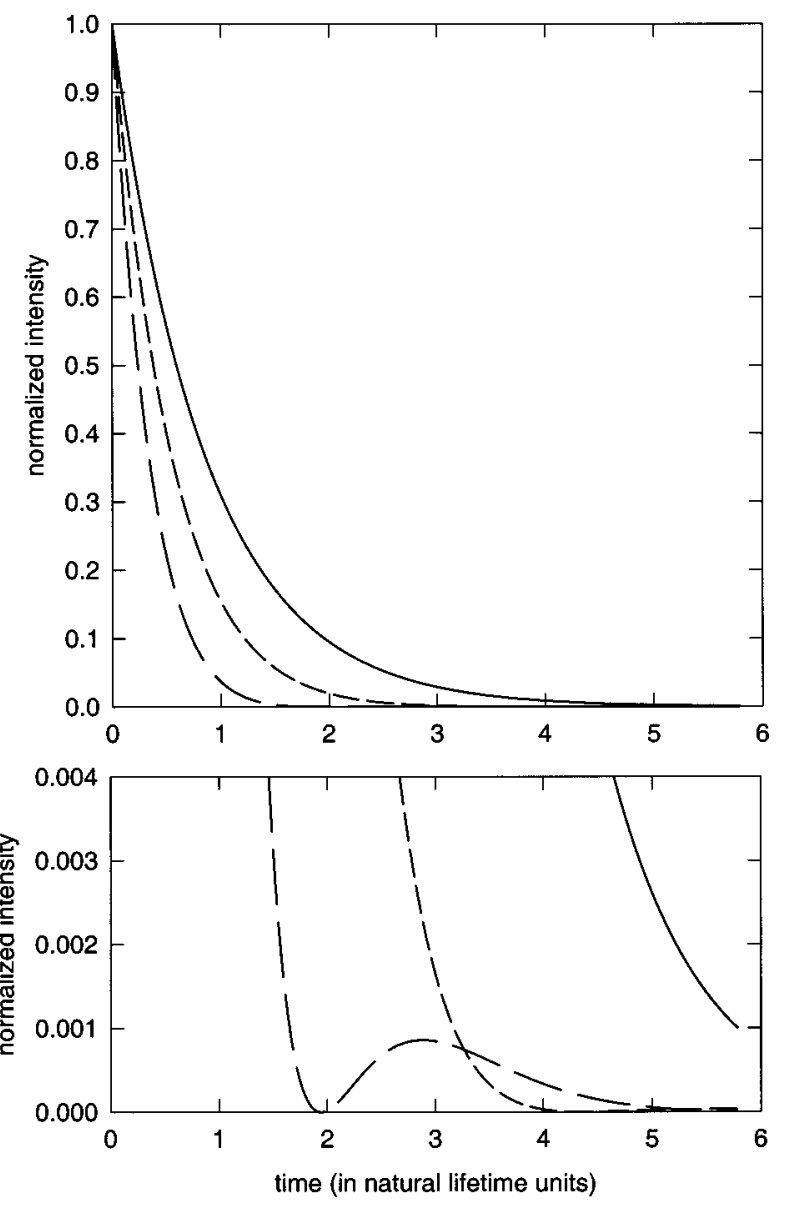

FIG. 1. The time-dependent forward scattering intensity for three different values of the sample thickness, i.e., effective number of resonant nuclei $N=5$ (solid line), 20 (shorter dashed line), and 45 (longer dashed line), is shown. Time is measured in units of the natural lifetime. Notice the "speedup" effect and, in the lower portion of the figure, the "dynamical beat." The intensity for each case is normalized to 1 at time $t^{\prime}=0$.

effect has been termed "dynamical beating.", 2 To see this more clearly the lower portion of Fig. 1 shows the results on an expanded scale. In principle, as the sample becomes even thicker, more local maxima appear in the time-dependent intensity curve. These results agree with those found earlier, both theoretically ${ }^{11}$ and experimentally. ${ }^{19,20}$ The advantage of our approach is that it gives insights into the origin of speedup and dynamical beating effects.

In order to understand the cause of these effects consider the contributions to the final result from the various coherent "paths." Figure 2 shows four amplitudes [i.e., terms inside the absolute value portion of Eq. (26) multiplied, for convenience, by the exponential function]. These four processes are, the non-hop processes (the solid line), the one-hop processes (the shorter dashed line), the two-hop processes (the longer dashed line), and the three-hop processes (the dasheddot line) for an effective number of nuclei (planes) $N=45$. Notice, as mentioned above, that the one-hop-process amplitude is $180^{\circ}$ out of phase with the amplitude for the no-hop processes. This is the main cause of the speedup effect. No- 


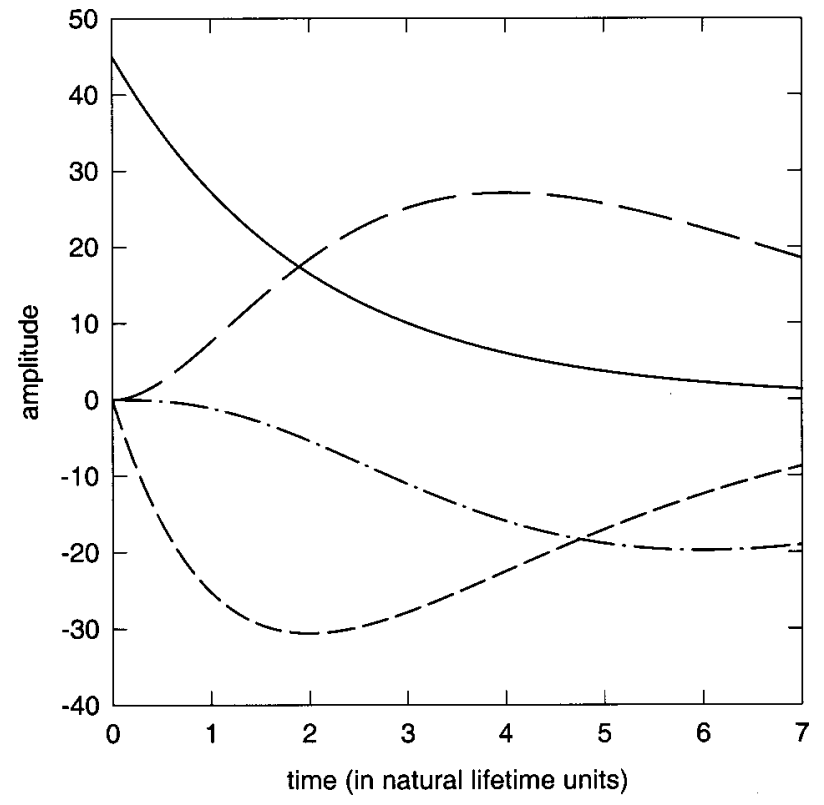

FIG. 2. The amplitudes of the no-hop (solid line), one-hop (shorter dashed line), two-hop (longer dashed line), and the threehop (dashed-dot line) processes are shown as a function of time in natural lifetime units. The amplitude axis shows the relative importance of each contribution. The case shown is for an effective number of nuclei $N=45$.

tice that when adding the one-hop to the no-hop amplitude the resulting curve goes toward zero faster than the no-hop exponential itself. Furthermore, for a sufficiently thick sample the two-hop amplitude at later times is larger in magnitude than the one-hop amplitude. This effect leads to the local maximum seen in Fig. 1 at time $t^{\prime}$ approximately equal to $3 \tau$ ( $\tau$ is the natural lifetime) and hence roughly accounts for the phenomenon of dynamical beats. Of course one must use the total expression, summing over all amplitudes, to obtain the precise result. Both speedup and dynamical beats effects have their origin in destructive and constructive interference between the amplitudes of the different coherent scattering paths. The even-numbered scattering paths give a positive contribution to the forward scattering amplitude, while the odd-numbered scattering paths give a negative contribution.

In principle, it should be possible to apply the coherent-path model to Bragg scattering. However, a difficulty arises when one considers the paths than need to be summed over. For the Bragg case, the number of paths over which the summation must be made becomes very large. An analytical method needs to be developed to overcome this difficulty.

\section{Quantum beats and polarization effects}

To treat quantum beats we need to consider situations in which the nuclei emit radiation from recoil-free transitions at two or more frequencies that have the same polarization. Under such conditions these frequencies will interfere producing quantum beats in the time-dependent

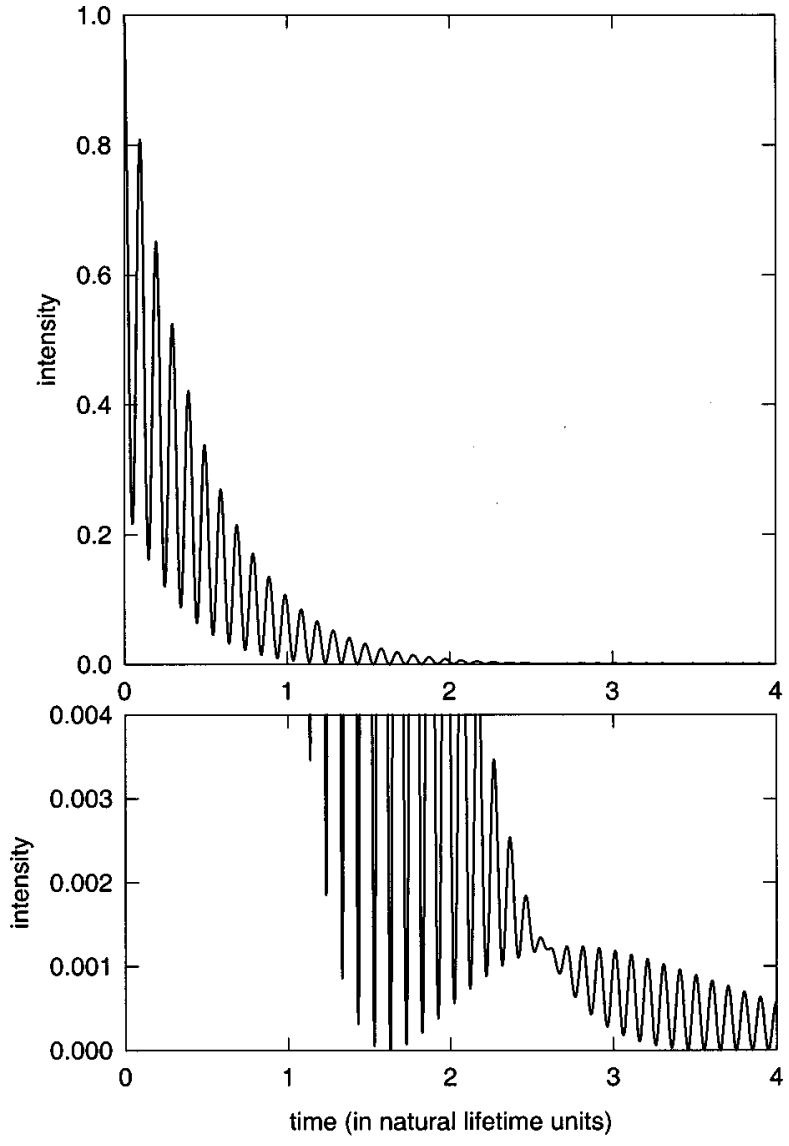

FIG. 3. Calculated results for the iron foil case when the magnetic field is in the direction of the synchrotron-radiation beam. Lines 1 and 4 have the same polarization. Lines 3 and 6 also have the same polarization but different from that of lines 1 and 4 . In the absence of an electric field gradient at the ${ }^{57} \mathrm{Fe}$ nuclei there is only one beat pattern corresponding to a beat period of about $14 \mathrm{nsec}$. These results are for the case when the effective number of resonant nuclei is $N=45$. The lower portion of the figure shows, on an expanded scale, the rather complicated structure in detail.

forward-scattering intensity. ${ }^{2,12,20,21}$ Of course polarization of the incident synchrotron radiation, as well as the polarization of the emitted radiation must be considered. $\Psi_{j}$ corresponds to the amplitude for emitting radiation at frequency $\omega_{j}$. Thus, if two or more transitions emit radiation in recoil-free processes at different frequencies having the same polarization, these amplitudes must be added due to their coherence before the intensity is calculated. This leads to the well-known phenomena of quantum beats.

The counting rate at the detector assuming that all paths are coherent is given by

$$
I\left(z, t^{\prime}\right)=c\left|\sum_{j} \Psi_{j}\left(z, t^{\prime}\right)\right|^{2}
$$

where the sum over $j$ is taken for those transitions that produce radiation having the same polarization. Introducing the wave packet of Eq. (24) into Eq. (29) gives 


$$
\begin{aligned}
I\left(z, t^{\prime}\right)= & \frac{\pi \gamma_{R}^{2}}{2 \hbar^{2} \Delta \omega_{p}} \mid \sum_{j} f_{j} e^{-i\left(\omega_{j}-i(\Gamma / 2 \hbar)\right) t^{\prime}} \\
& \times\left.\left[N+\sum_{n=1}^{N-1}\left(\frac{-f_{j} \gamma_{R}}{2 \hbar}\right)^{n}\left(\begin{array}{c}
N \\
n+1
\end{array}\right) \frac{t^{\prime n}}{n !}\right]\right|^{2},
\end{aligned}
$$

where an $f_{j}$ has been inserted to account for the specific transition probability.

In order to be specific, consider the synchrotron radiation interacting with an iron foil which is polarized so that the internal magnetic field is in the same direction as the synchrotron beam. The synchrotron radiation is almost completely linearly polarized in the plane of the synchrotron ring. This polarization can be expressed in terms of a superposition of right and left circularly polarized states. For the case under discussion the emitted radiation is composed of only right and left circularly polarized components also. If one labels the transitions from 1 to 6 according to their increasing frequencies $\omega_{i}(i=1-6)$, then line 1 and line 4 will have one polarization while line 3 and line 6 will have the other. Assuming no electric-field gradient at the sites of the ${ }^{57} \mathrm{Fe}$ nuclei, the difference in each pair of frequencies is the same. Thus there will be only one beat frequency corresponding to about 14 nsec appearing in the time-dependent intensity spectrum. As mentioned above, one needs to incorporate the transition probability for each transition considered. In this case it is well known that lines 1 and 6 have relative intensities of 0.75 and lines 3 and 4 have relative intensities of 0.25 . The resulting time-dependent forward scattering intensity is then given by Eq. (31) specialized for this case

$$
I\left(t^{\prime}\right)=\frac{\pi \gamma_{R}^{2}}{2 \hbar^{2} \Delta \omega_{p}}\left|\frac{3 f}{8} e^{-i\left(\omega_{1}-i(\Gamma / 2 \hbar)\right) t^{\prime}} \sum_{n=0}^{N-1}\left(\begin{array}{c}
N \\
n+1
\end{array}\right)\left(\frac{3 f \gamma_{R} t^{\prime}}{8 \hbar}\right)^{n} \frac{(-1)^{n}}{n !}+\frac{f}{8} e^{-i\left(\omega_{4}-i(\Gamma / 2 \hbar)\right) t^{\prime}} \sum_{n=0}^{N-1}\left(\begin{array}{c}
N \\
n+1
\end{array}\right)\left(\frac{f \gamma_{R} t^{\prime}}{8 \hbar}\right)^{n} \frac{(-1)^{n}}{n !}\right|^{2}
$$

Figure 3 gives an example of the quantum-beat effect. In Fig. 3 the result is calculated for the case when $N=45$. This result agrees precisely with that obtained using $^{18}$ the classical optical model. The time dependence of the forward-scattered intensity is complicated here because of the combination of effects due to quantum beats [the factors $e^{ \pm i\left(\omega_{1}-\omega_{4}\right) t}$ in Eq. (31)] and to speedup and dynamical beats (the sum over $n$, which reflects the multiple scattering, as has been discussed in the previous section).

\section{B. Incoherent processes}

\section{General remarks}

The coherent-path model can also be used to study incoherent processes. In general, when using polycrystalline matter, we could term an incoherent process as any process that produces radiation that does not travel in the forward direction. However, a fraction of the incoherent radiation may go forward and produce a background for the coherent forwardscattered radiation. Thus, it is useful to know this background even when radiation in the forward direction has to be analyzed.

Several experiments have been performed that deal with the incoherent scattering of SR itself. The Brookhaven group $^{22}$ has studied the time evolution of coherent and incoherent scattering of SR from a ${ }^{57} \mathrm{Fe}$ foil. The phonon spectrum of $\alpha$-iron ${ }^{23}$ and of $\alpha$-iron and other iron compounds ${ }^{24}$ has been obtained by the observation of nuclear-resonant fluorescence versus the energy of incident SR. The phonon density of states can be deduced from these measurements. Analogous studies have been performed on other iron compounds. $^{25}$ The same authors ${ }^{26}$ have observed single- nucleus quantum beats in the incoherent scattering of SR when $\alpha$-iron was excited far from the resonance energy, thus accompanied by phonon creation or annihilation. These single-nucleus quantum beats are well known from timedependent perturbed angular correlation measurements. The temperature dependence of nuclear recoil and of the density of phonon states in iron has also been studied. ${ }^{27}$ Another incoherent channel that can be studied is the delayed emission of conversion electrons that follows the excitation. This has been done by the Argonne team ${ }^{28}$ in the case of an iron foil. Several other references deal with incoherent scattering (see Refs. 29-31). A two-wave diffraction theory exists ${ }^{32}$ that deals with inelastic coherent scattering of SR in perfect crystals

We will now discuss how the present approach can be used to study such incoherent processes. Recall that in the model there is a chain of $N$ effective nuclei or, equivalently a stack of $N$ effective planes. In Ref. 12 Sturhahn and Gerdau employ the "trick" of cutting up the crystal into thin platelets in order to calculate their final results. The number of thin platelets does not appear in their solution. It is only the resonant thickness of the sample that appears in the result. In our case the effective $N$ platelets arise naturally as part of the model itself and the factor $N$ is the only parameter in our final result. Thus, for these incoherent processes we consider that the actual sample can be modeled to consist of $N$ planes located at the positions of the $N$ effective nuclei. Suppose we focus our attention on the nucleus labeled $n$ in the model. This is the nucleus, located at $z_{n}$, i.e., located at the $n$th plane, which is assumed to emit the incoherent radiation. This incoherent radiation could be due to, among other possibilities, internal conversion. The conversion electrons can 
be detected directly, or indirectly using the resulting $\mathrm{x}$ rays. Another source of incoherent radiation is emission with recoil. The analysis of this recoil process merits some extra attention. (The analysis of processes with recoil will be done in the next section.)

It will be shown below that the intensity of the radiation corresponding to incoherent processes has a complicated time structure. First one has to consider the intensity corresponding to incoherent radiation coming from the $n$th plane in the "sample." The total incoherent intensity will then be the sum of the individual intensities from each plane. This is so because, in this case, there is no interference between the incoherent radiation coming from each effective plane. However, in order to calculate the intensity coming from the $n$th plane, one has to consider all coherent paths that lead to the excitation of the effective nucleus that represents that $n$th plane. The intensity that results from a final incoherent emission from the $n$th plane is then given by a modification of Eq. (26). One has the intensity from the $n$th nucleus (plane) as

$$
I_{\mathrm{inc}, n}\left(t^{\prime}\right)=C_{\mathrm{inc}} e^{-(\Gamma / \hbar) t^{\prime}}\left|\sum_{m=0}^{n-1}\left(\begin{array}{c}
n-1 \\
m
\end{array}\right)\left(-\frac{f \gamma_{R} t^{\prime}}{2 \hbar}\right)^{m} \frac{1}{m !}\right|^{2} .
$$

Here the constant $C_{\text {inc }}$ depends on the nature of the incoherent process. The total time-dependent intensity due to an incoherent process is then given by

$$
I_{\text {inc,total }}\left(t^{\prime}\right)=\sum_{n=1}^{N} I_{\text {inc, } n}\left(t^{\prime}\right) .
$$

In the following, processes with recoil and conversionelectron processes will be discussed in more detail.

\section{Processes with recoil}

The recoil of a nucleus emitting radiation can be expressed in terms of the normal modes of the lattice, i.e., in terms of phonon creation. The vibrational states are nonlocalized, so the decay with recoil is spatially coherent. However, because of the quasicontinuum of phonon states, there is no coherent enhancement to these decay modes. In principle there are quantum beats between processes with and without recoil. In practical experience however, these beats are not observed because the interference terms, giving rise to beats, vanish. This is due to the fact that the beat frequencies form a quasi-continuum which implies that, when averaging, the interference terms will disappear. Even when an isolated phonon frequency is excited due to recoil (e.g., due to the recoil of an oversized impurity in a lattice), the quantum beats between processes with and without recoil cannot be observed. This is due to the extremely high beat frequency in this case. The present experimental techniques do not allow for a sufficient time resolution.

If we denote the recoil fraction as $(1-f)$, it is possible to express the recoil processes in the following way. First we consider each effective nucleus (plane) in our sample separately. Again we focus our attention on the $n$th plane (nucleus) in our sample: this is the plane (nucleus) that will emit radiation and, in the process, recoil. This radiation is now off resonance and will not be resonantly absorbed even if the radiation must pass through additional material in going to the detector. Now this nucleus, or plane, is located at $z_{n}$. The problem now is to consider all coherent paths that lead to excitation of that nucleus (plane). The intensity of such a process that results in a final emission with recoil from the $n$th nucleus (plane) (recall that the "final" nucleus in a "path" radiates radiation directly to the detector) is given by the modification of Eq. (32). In order to obtain the desired expression one simply needs to include all indistinguishable paths that lead to the excitation of the $n$th nucleus (plane) in the sample. This nucleus is then assumed to recoil as it emits radiation to the detector. For such a process one has the intensity

$$
\begin{aligned}
I_{(1-f), n}\left(t^{\prime}\right)= & \frac{\pi \gamma_{R}^{2}}{2 \hbar^{2} \Delta \omega_{p}}(1-f) f e^{-(\Gamma / \hbar) t^{\prime}} \\
& \times\left|\sum_{m=0}^{n-1}\left(\begin{array}{c}
n-1 \\
m
\end{array}\right)\left(-\frac{f \gamma_{R} t^{\prime}}{2 \hbar}\right)^{m} \frac{1}{m !}\right|^{2} .
\end{aligned}
$$

It is of course possible for all nuclei (planes) in our sample to do similarly. With the discussion at the beginning of this section, it is obvious that the intensities of radiation due to each nucleus (plane) can be added together to obtain the final recoil-process result. The time-dependent intensity due to processes with recoil is then given by

$$
I_{(1-f), \text { total }}\left(t^{\prime}\right)=\sum_{n=1}^{N} I_{(1-f), n}\left(t^{\prime}\right) .
$$

Notice, however, that in our discussion electronic absorption has been neglected. Depending on the specific sample and placement of the detector relative to the sample it is possible that the radiation from the $n$th nucleus (plane) is attenuated more or less than the radiation from the $m$ th nucleus (plane) due to electronic absorption.

For forward-scattering measurements the total result from this recoil process must be added to the intensity from the recoil-free processes, since radiation from the recoil processes may also reach the detector. However, this contribution should be small because the incoherent radiation goes into all $4 \pi$ steradians. On the other hand, for radiation in the nonforward direction, this contribution should be dominant in the time-delayed portion of the spectrum. Figure 4 shows calculated examples of the temporal shape due to the incoherent processes alone, neglecting electronic absorption. We have considered three samples of differing thicknesses. They correspond to thicknesses represented by an effective number of planes (nuclei): $\quad N=5,20$, and 45 . It is of interest to note that in the data analysis of the time evolution of incoherent nuclear scattering by Bergmann et al. ${ }^{22}$ using the semiclassical optical model, they were forced to consider the contributions resulting from dividing their sample into planes. The final fit to the data was made by assuming that the spectrum consisted of only two components. The two components they choose were; an averaged speedup component, and a component having the normal lifetime. In our 


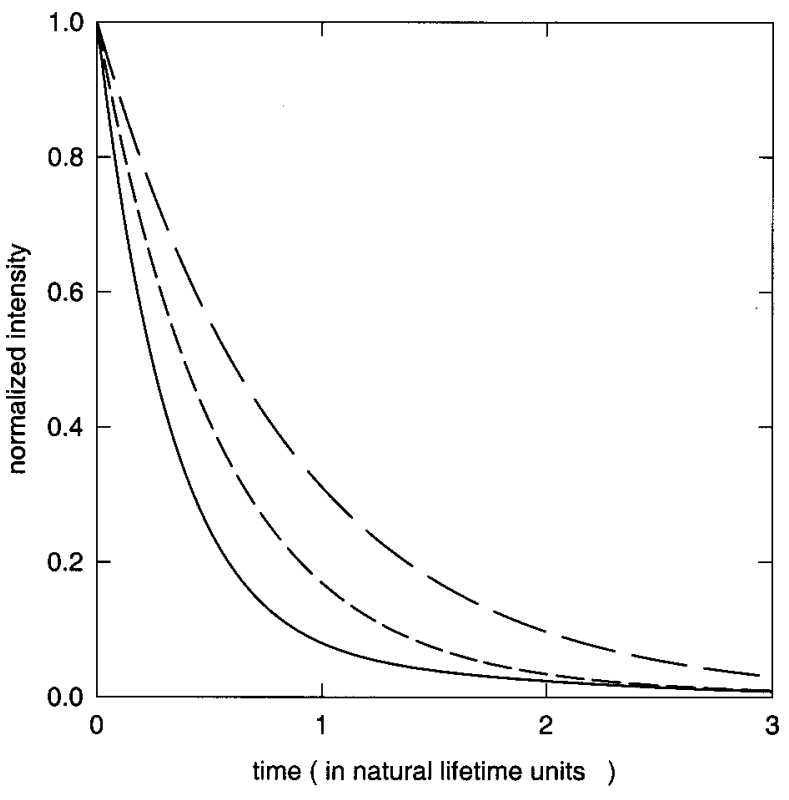

FIG. 4. The time-dependent intensity, due to the processes that happen with recoil (incoherent processes), are shown. The calculated curves are for "samples" of three different thicknesses $(N)$. One sample has $N=45$ (the solid line), another has $N=20$ (the shorter dashed line), and the last has $N=5$ (the longer dashed line). Each of the curves has been normalized to one at time $t^{\prime}=0$. See the text for a discussion of these calculations.

model the spectrum would consist of $N$ components. Each component has its own speedup contribution ranging from a no speedup component, due to the sample plane farthest upstream, to a maximum speedup component resulting from the plane farthest down-stream. The general trend of the experimental data ${ }^{22}$ appears to agree with the new model. Figure 4 shows some expected results for the time-dependent intensity due to the incoherent channel, i.e., detection of radiation from recoil events. However, a detailed analysis of the data is needed to see if the new model gives a better fit. Such a detailed analysis requires knowledge of the exact experimental conditions.

\section{Conversion-electron processes}

The conversion electron or the resulting $\mathrm{x}$ rays can be detected, as a function of time after the synchrotron radiation pulse, in addition to the processes already discussed. From the coherent-path model the resulting time-dependent intensity can be calculated rather easily. In fact the shape of the time-dependent intensity will be similar to that of the recoilprocess time-dependent intensity. One difference is due to the difference between the radiative width and the conversion-electron width. Moreover, the conversion electrons, or perhaps the resulting rather low-energy $\mathrm{x}$ rays, can not travel very far in the material. For these cases it is quite important to consider the fact that the contributions from each "nucleus" in the "sample" may be strongly attenuated depending on the distance the conversion electron or $\mathrm{x}$ ray must travel in the material before reaching the detector. In the case of conversion-electron detection only the nuclei close to the surface of the sample nearest to the detector will contribute significantly, thus modifying the shape of the time-dependent intensity from that shown in Fig. 4. This surface-plane effect is familiar from ordinary conversionelectron Mössbauer spectroscopy. The time-dependent electron emission from ${ }^{57} \mathrm{Fe}$ nuclei has been observed by Sturhahn et al. $^{28}$ Such experimental results can be compared with this model's predictions, but close collaboration is again required in order to be aware of the needed experimental details.

\section{CONCLUSIONS}

One may very well ask how is it that this apparently "one-dimensional" model gives results that are in such good agreement with experiment and previous theory. In fact the model is not really a one-dimensional theory, as noted above. This can be seen as follows. Recall that the resonant gamma radiation is treated as a plane wave, and the phase shift of the forward-scattered radiation due to a single effective nucleus is $\pi$. In x-ray diffraction ${ }^{33}$ it is well known that a single resonant scattering gives a $\pi / 2$ phase shift and a further $\pi / 2$ phase shift arises when a summation is made over the whole plane of resonant scatterers. This result is also noted in Ref. 34 . Notice that the model gives such a $\pi$ phase shift, when scattering off a single "effective" nucleus, as seen by the minus sign in Eq. (26). Thus the theory more appropriately corresponds to a nuclear resonant sample represented by $N$ "effective" parallel planes or slices. This realization helps explain why the model works so well.

The model gives a microscopic picture of nuclearresonant forward scattering of synchrotron radiation from polycrystalline matter containing resonant nuclei. A closedform solution is obtained consisting of a finite series. The number of terms in the solution depends on the "thickness", $(N)$ of the sample. Each term in the solution corresponds to a scattering sequence or path the radiation takes in reaching the detector. The resulting equations are easy to use and the necessary calculations are quite simple to perform.

The model gives a clear physical explanation of both the speedup and dynamical-beat effects that have already been observed experimentally. These effects arise naturally as a consequence of summing over the interfering amplitudes corresponding to each possible path the radiation takes on getting to the detector. In order to simplify the language we say that when the radiation from one nucleus (plane) is absorbed and reradiated by another nucleus (plane), the radiation "hops" on that other nucleus (plane). The origin of the speedup effect is primarily due to the $180^{\circ}$ phase shift of the "'one-hop" amplitude as compared to the "no-hop" amplitude. In the one-hop process SR radiation that has been resonantly absorbed by one nucleus (plane) is then absorbed and reemitted, in a recoil-free fashion, by another nucleus (plane) before the radiation reaches the detector. The "no-hop" process corresponds to the case when the SR radiation has simply been resonantly absorbed and re-emitted to the detector by the same nucleus (plane). The resulting negative sign in the one-hop contribution leads to a subtraction from the ordinary exponential temporal decay, due to radiation from a single decaying nucleus (plane), and hence leads to the speedup effect early in the decay. Similarly, radiation that 
has been resonantly absorbed and reemitted in a "two-hop" process is back in phase with the radiation that has been resonantly absorbed and re-emitted by a single nucleus (plane), i.e., the no-hop case. When the sample is sufficiently thick this two-hop amplitude is larger in magnitude than the one-hop amplitude, at later times, and hence leads to the observed dynamical beats. Of course all multihop coherent paths must be summed over to obtain the final result. The amplitudes corresponding to the odd-numbered scattering paths, i.e., odd numbered "hops," are $180^{\circ}$ out of phase with the amplitudes corresponding to the even-numbered scattering paths. This phenomenon is responsible for the complex beat pattern observed for very thick samples.

The model also shows why the speedup effect is more pronounced using a radioactive source ${ }^{16}$ then when using synchrotron radiation as the source. For the synchrotronradiation case there is a term in the calculation in which $N$ nuclei (planes) radiate directly to the detector at their normal decay rate thus weighting the normal decay rate significantly. There is no such term in the radioactive-source case.

Various incoherent processes can also be analyzed using this model. Such processes include those in which the nuclei recoil when emitting radiation or emit conversion electrons instead of a gamma ray. Radiation produced, when a resonant nucleus emits radiation and in the process recoils, may be recorded in the detector. This contribution should be relatively small, in the forward direction, since this radiation is emitted into the whole $4 \pi$ solid angle, and hence can be observed most effectively by looking in nonforward directions.

The understanding of the behavior of the scattered intensity in a nonforward direction is important because several experiments have already been performed to study these incoherent processes, as noted above. Furthermore it is to be expected that additional experiments will be done in the future.

It appears that this new model, because of its simplicity, can be utilized in the future to treat other more complicated cases. A theoretical treatment ${ }^{29}$ of the diffusion problem has already been given using the semiclassical optical model. In addition, time domain experimental results ${ }^{35}$ in the presence of diffusion have been obtained. Furthermore, single-nucleus quantum beats ${ }^{26}$ excited by synchrotron radiation have also been observed. For these experiments it is crucial that the collective coherent state is not produced in the scatterer. This condition was established by detecting radiation at $90^{\circ}$ from the forward direction and setting the incident energy $20 \mathrm{meV}$ above the nuclear-resonant energy. This experiment has shown that it may be possible to simultaneously investigate coherent and incoherent scattering, and in the process sort out the absorption and emission processes occurring with and without recoil. Thus there are many interesting and informative experiments that can be done with the advent of the third generation synchrotrons when the beam-line experimental stations include low temperature facilities as well as the ability to apply various external fields. The theoretical model presented here should prove useful in understanding such future experiments.

\section{ACKNOWLEDGMENTS}

This work was supported in part by the IAP-program P407, financed by the Belgian Federal Office for Scientific, Technical and Cultural Affairs, and by the Fonds voor Wetenschappelijk Onderzoek Vlaanderen.

\section{APPENDIX A}

In this appendix it will be shown how $B_{m, j}(\omega)$ can be calculated starting from Eq. (23)

$$
\begin{aligned}
\left(\omega-\omega_{j}+i \frac{\gamma_{c}}{2 \hbar}\right) B_{m, j}(\omega) \\
=A \sum_{k} \frac{V_{k, j}}{\hbar} e^{i k z_{m}}+\sum_{k^{\prime}} \sum_{m^{\prime}} B_{m^{\prime}, j} \frac{\left|V_{k^{\prime}, j}\right|^{2}}{\hbar^{2}} \\
\quad \times e^{i k^{\prime}}\left(z_{m}-z_{m^{\prime}}\right) \frac{1}{\omega-\omega_{k^{\prime}}+i \varepsilon} .
\end{aligned}
$$

The last term in Eq. (A1) can be divided into two parts; one with $m^{\prime} \neq m$, and the other $m^{\prime}=m$. Then

$$
\begin{aligned}
\left(\omega-\omega_{j}+i \frac{\gamma_{c}}{2 \hbar}\right) B_{m, j}(\omega) \\
=B_{m, j}(\omega) \sum_{k^{\prime}} \frac{\left|V_{k^{\prime}, j}\right|^{2}}{\hbar^{2}\left(\omega-\omega_{k^{\prime}}+i \varepsilon\right)}+A \sum_{k} \frac{V_{k, j} e^{i k z_{m}}}{\hbar} \\
\quad+\sum_{k^{\prime}} \sum_{m^{\prime} \neq m} B_{m^{\prime}, j}(\omega) \frac{\left|V_{k^{\prime}, j}\right|^{2} e^{i k^{\prime}\left(z_{m}-z_{m^{\prime}}\right)}}{\hbar^{2}\left(\omega-\omega_{k^{\prime}}+i \varepsilon\right)} .
\end{aligned}
$$

Considering the first term on the right-hand side, the sum over $k^{\prime}$ can be converted to an integral using the prescription $^{14}$

$$
\frac{2 \pi}{L} \sum_{k^{\prime}} \rightarrow \int_{-\infty}^{\infty} d k^{\prime}
$$

where $L$ is a normalizing length which appears when sums on the discrete variable $k^{\prime}$ are transformed into integrals in one dimension.

Again using the symbolic identity ${ }^{14}$ we have

$$
\frac{1}{\omega-\omega_{k^{\prime}}+i \varepsilon}=P \frac{1}{\omega-\omega_{k^{\prime}}}-i \pi \delta\left(\omega-\omega_{k^{\prime}}\right),
$$

where $P$ represents the principal part of the involved integral, the first series of Eq. (A2) can be calculated. This results in a principal value term and a delta function term. The principal value term corresponds to a frequency shift when brought to the left-hand side. This will be neglected. The delta function term corresponds to a radiative width $\gamma_{R}$ where

$$
\gamma_{R}=\frac{2 L}{\hbar c}\left|V_{k^{\prime}, j}(\omega)\right|^{2} .
$$


As in three dimensions, the presence of $L$ in this and in the following expressions is only apparent, because the matrix elements such as $\left|V_{k^{\prime}, j}\right|^{2}$ contain $1 / L$. Collecting terms on the left-hand side gives

$$
\begin{aligned}
& \left(\omega-\omega_{j}+i \frac{\Gamma}{2 \hbar}\right) B_{m, j}(\omega) \\
& \quad=A \sum_{k} \frac{V_{k, j} e^{i k z_{m}}}{\hbar}+\sum_{m^{\prime} \neq m} B_{m^{\prime}, j} \sum_{k^{\prime}} \frac{\left|V_{k^{\prime}, j}\right|^{2} e^{i k^{\prime}\left(z_{m}-z_{m^{\prime}}\right)}}{\hbar^{2}\left(\omega-\omega_{k^{\prime}}+i \varepsilon\right)},
\end{aligned}
$$

where $\Gamma$ is the total width equal to the sum of the conversion-electron and radiative widths. The sum over $k$, in the first term on the right-hand side, can be converted into an integral and evaluated. The result is

$$
A \sum_{k} V_{k, j} \frac{e^{i k z_{m}}}{\hbar} \Rightarrow \frac{L}{2 \pi} \frac{\Delta \omega_{p}}{c} \frac{V_{k_{0}, j}}{\hbar} e^{i k_{0} z_{m}}
$$

where $\Delta \omega_{p}$ is the effective band-width of the synchrotron radiation pulse, $k_{0}$ is the central wave number in the frequency range of interest, and noting that $V_{k, j}$ is not a strong function of $k$. Substituting into Eq. (A6) and applying Jordan's lemma to the integral resulting from the sum over $k^{\prime}$ gives

$$
\begin{aligned}
\left(\omega-\omega_{j}+i \frac{\Gamma}{2 \hbar}\right) B_{m, j}(\omega) & \\
= & \frac{L A \Delta \omega_{p} V_{k_{0}, j}}{2 \pi c \hbar} e^{i k_{0} z_{m}} \\
& -i \frac{L}{\hbar^{2} c}\left|V_{k^{\prime}, j}(\omega)\right|^{2} \sum_{m^{\prime}<m} B_{m^{\prime}, j}(\omega) e^{i(\omega / c)\left(z_{m}-z_{m^{\prime}}\right)}
\end{aligned}
$$

where $z_{m}>0$ and $z_{m}>z_{m^{\prime}}$.

Equation (A8) is a recursion relation relating the $B_{m, j}(\omega)^{\prime}$ s. It is not too difficult to show that

$$
B_{m, j}(\omega)=\frac{L A \Delta \omega_{p}}{2 \pi c} \frac{V_{k_{0}, j}}{\hbar}\left[\begin{array}{c}
\frac{e^{i k_{0} z_{m}}}{\left(\omega-\omega_{j}+i \frac{\Gamma}{2 \hbar}\right)} \\
+\sum_{n=1}^{m-1}\left(\frac{-i \gamma_{R}}{2 \hbar}\right)^{n} \frac{1}{\left(\omega-\omega_{j}+i \frac{\Gamma}{2 \hbar}\right)^{n+1}} \sum_{n^{\prime}=1}^{m-n}\left(\begin{array}{c}
m-1-n^{\prime} \\
n-1
\end{array}\right) e^{i k_{0} z_{m^{\prime}} e^{i(\omega / c)\left(z_{m}-z_{m^{\prime}}\right)}}
\end{array}\right],
$$

where the factor $\left(m-1-n^{\prime}\right)$ over $(n-1)$ is the binomial coefficient and $\gamma_{R}$ has been inserted into the equation.

\section{APPENDIX B}

In Appendix B the outgoing wave packet is calculated. From Eq. (22), $C_{k^{\prime}}(\omega)$ can be expressed in terms of $B_{m, j}(\omega)$, and when the value of $B_{m, j}(\omega)$ from Eq. (A9) is inserted, the following expression is obtained:

$$
\begin{aligned}
C_{k^{\prime}}(\omega)= & \frac{L A \Delta \omega_{p}}{2 \pi c} \frac{V_{k_{0}, j} V_{k^{\prime}, j}^{*}}{\hbar^{2}} \sum_{m=1}^{N} \frac{e^{i k^{\prime} z_{m}}}{\left(\omega-\omega_{k^{\prime}}+i \varepsilon\right)} \\
& \times\left[\begin{array}{l}
\frac{e^{i k_{0} z_{m}}}{\left(\omega-\omega_{j}+i \frac{\Gamma}{2 \hbar}\right)} \\
+\sum_{n=1}^{m-1}\left(\frac{-i \gamma_{R}}{2 \hbar}\right)^{n} \frac{1}{\left(\omega-\omega_{j}+i \frac{\Gamma}{2 \hbar}\right)^{n+1}} \sum_{n^{\prime}=1}^{m-n}\left(\begin{array}{c}
m-1-n^{\prime} \\
n-1
\end{array}\right) e^{i k_{0} z_{m^{\prime}}} e^{i(\omega / c)\left(z_{m}-z_{m^{\prime}}\right)}
\end{array}\right] .
\end{aligned}
$$

Next one needs to take the Fourier transform of $C_{k^{\prime}}(\omega)$, conveniently defined here [see Eq. (3)] as

$$
c_{k^{\prime}}=-\frac{1}{2 \pi i} \int_{-\infty}^{\infty} d \omega C_{k^{\prime}}(\omega) e^{i\left(\omega_{k^{\prime}}-\omega\right) t}
$$

The only pole in these integrals that contributes to the final answer is the one at $\omega=\omega_{k^{\prime}}-i \varepsilon$. The other poles of Eq. (B1) give a zero contribution in the resulting plane-wave packet describing the outgoing radiation [Eq. (B4) below]. This is a consequence of the situation in which the detector is positioned at $z$ which is beyond the positions of all the nuclei located at the $z_{m}$ 's. Using this observation, one can obtain $c_{k^{\prime}}$, 


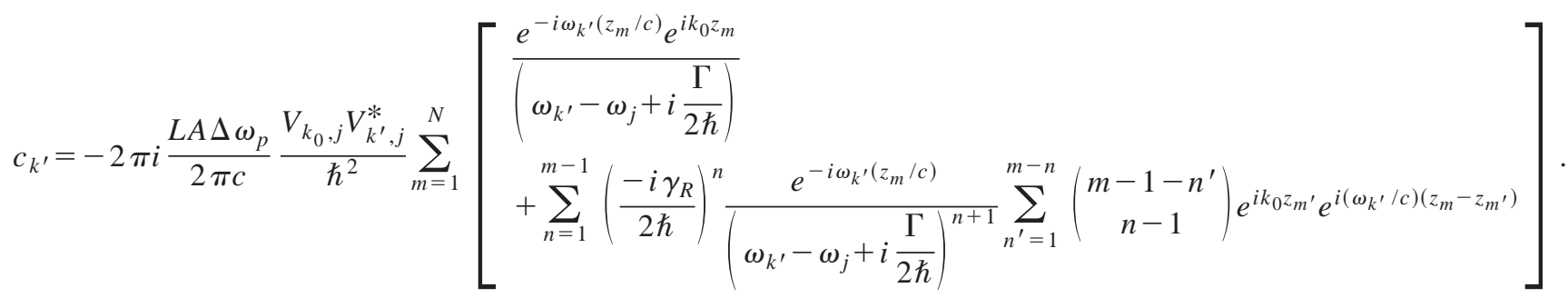

The next step in the calculation is to construct the resulting plane-wave packet that describes the radiation emitted by the nuclei according to the form

$$
\Psi_{j}(z, t)=\frac{\sqrt{L}}{2 \pi c} \int_{-\infty}^{\infty} d \omega_{k^{\prime}} e^{i \omega_{k^{\prime}}(z / c-t)} c_{k^{\prime}} .
$$

Equation (B3) for $c_{k^{\prime}}$ shows that there are two contributions that need to be evaluated. They are

$$
c_{k^{\prime} 1}=-2 \pi i \frac{L A \Delta \omega_{p}}{2 \pi c} \frac{V_{k_{0}, j} V_{k^{\prime}, j}^{*}}{\hbar^{2}} \sum_{m=1}^{N} \frac{e^{-i \omega_{k^{\prime}}\left(z_{m} / c\right)} e^{i k_{0} z_{m}}}{\left(\omega_{k^{\prime}}-\omega_{j}+i \frac{\Gamma}{2 \hbar}\right)}
$$

and

$$
\begin{aligned}
c_{k^{\prime}, 2}= & -2 \pi i \frac{L A \Delta \omega_{p}}{2 \pi c} \frac{V_{k_{0}, j} V_{k^{\prime}, j}^{*}}{\hbar^{2}} \\
& \times \sum_{m=1}^{N} \sum_{n=1}^{m-1}\left(\frac{-i \gamma_{R}}{2 \hbar}\right)^{n} \frac{e^{-\omega_{k^{\prime}}\left(z_{m} / c\right)}}{\left(\omega_{k^{\prime}}-\omega_{j}+i \frac{\Gamma}{2 \hbar}\right)^{n+1}} \\
& \times \sum_{n^{\prime}=1}^{m-n}\left(\begin{array}{c}
m-1-n^{\prime} \\
n-1
\end{array}\right) e^{i k_{o^{2}} z^{\prime}} e^{i\left(\omega_{k^{\prime}} / c\right)\left(z_{m}-z_{n^{\prime}}\right)} .
\end{aligned}
$$

The result for $c_{k^{\prime} 1}$ is found by substituting its value from Eq. (B5) into Eq. (B4)

$$
\begin{aligned}
\Psi_{1 j}(z, t)= & \frac{-\sqrt{L} L A \Delta \omega_{p}}{c^{2}} \frac{V_{k_{0}, j} V^{*}\left(\omega_{j}\right)}{\hbar^{2}} \\
& \times N e^{-i\left(\omega_{j}-i(\Gamma / 2 \hbar)\right)(t-z / c)},
\end{aligned}
$$

where $e^{i k_{0} z_{m}} \times e^{-i \omega_{j}\left(z_{m} / c\right)}$ essentially gives 1 , and $e^{-\left(\Gamma z_{m} / 2 \hbar c\right)} \approx 1$. The result for $c_{k^{\prime} 2}$ is found from Eqs. (B6) and (B7)

$$
\begin{aligned}
\Psi_{2 j}(z, t)= & \frac{-\sqrt{L} L A \Delta \omega_{p}}{c^{2}} \frac{V_{k_{0}, j} V^{*}\left(\omega_{j}\right)}{\hbar^{2}} \\
& \times e^{-i\left(\omega_{j}-i(\Gamma / 2 \hbar)(t-z / c)\right.} \\
& \times \sum_{n=1}^{N-1}\left(\frac{-\gamma_{R}}{2 \hbar}\right)^{n}\left(\begin{array}{c}
N \\
n+1
\end{array}\right) \frac{\left(t-\frac{z}{c}\right)^{n}}{n !},
\end{aligned}
$$

where $t-\left(z-z_{n}\right) / c \cong t-z / c$ and the approximations used above also apply. The total result is

$$
\begin{aligned}
\Psi_{j}\left(z, t^{\prime}\right)= & \frac{-\sqrt{L} L A \Delta \omega}{c^{2}} \frac{V_{k_{0}, j} V^{*}\left(\omega_{j}\right)}{\hbar^{2}} e^{-i\left(\omega_{j}-i(\Gamma / 2 \hbar)\right) t^{\prime}} \\
& \times\left[N+\sum_{n=1}^{N-1}\left(\frac{-\gamma_{R}}{2 \hbar}\right)^{n}\left(\begin{array}{c}
N \\
n+1
\end{array}\right) \frac{t^{\prime n}}{n !}\right]
\end{aligned}
$$

where $t^{\prime}=t-z / c$.

This can be transformed further, using a simple model for the synchrotron radiation pulse. For a constant value $A_{k}(\omega)$ $=A$ one has

$$
a_{k}(t)=i A \delta(t) e^{i \omega_{k} t}
$$

The synchrotron pulse has a certain finite frequency width, approximately equal to the inverse of the duration of the pulse, $\delta(t)$ can be approximated by $\Delta \omega_{p}$ in the interval $\left[-1 / 2 \Delta \omega_{p}, 1 / 2 \Delta \omega_{p}\right]$. Elsewhere the function is assumed to be zero. Then

$$
a_{k}(t)=i A \Delta \omega_{p} e^{i \omega_{k} t}
$$

for $t$ close to zero. For other times $a_{k}(t)=0$.

The normalization of the SR state for $t$ close to zero gives

$$
\sum_{k}\left|a_{k}\right|^{2}=\frac{L}{2 \pi} \int_{k_{0}-\Delta / 2}^{K_{0}+\Delta / 2}\left|a_{k}\right|^{2} d k=1
$$

Substituting Eq. (B11) into Eq. (B12) gives after integration

$$
\frac{L}{2 \pi}|A|^{2} \Delta \omega_{p}^{2} \Delta=1
$$

With $\Delta=\Delta \omega_{p} / c$ one has, if $A$ is taken as real (which is not essential) 


$$
A=\sqrt{\frac{2 \pi c}{L \Delta \omega_{p}}} \frac{1}{\Delta \omega_{p}} .
$$

Putting Eq. (B14) into Eq. (B9) gives finally

$$
\Psi_{j}\left(z, t^{\prime}\right)=-\frac{L}{c^{2}} \sqrt{\frac{2 \pi c}{\Delta \omega_{p}}} \frac{V_{k_{0}, j} V^{*}\left(\omega_{j}\right)}{\hbar^{2}} e^{-i\left(\omega_{j}-i(\Gamma / 2 \hbar)\right) t^{\prime}}\left[N+\sum_{n=1}^{N-1}\left(\frac{-\gamma_{R}}{2 \hbar}\right)^{n}\left(\begin{array}{c}
N \\
n+1
\end{array}\right) \frac{t^{\prime n}}{n !}\right] .
$$

As already has been mentioned before, the presence of $L$ is only apparent because $V_{k_{0}, j} V^{*}\left(\omega_{j}\right)$ contains $1 / L$. Equation (B15) corresponds to Eq. (24) of the main text.

${ }^{1}$ E. Gerdau, R. Rüffer, H. Winkler, W. Tolksdorf, C. P. Klages, and J. P. Hannon, Phys. Rev. Lett. 54, 835 (1985).

${ }^{2}$ G. V. Smirnov, Hyperfine Interact. 97/98, 551 (1996) and references cited therein.

${ }^{3}$ J. P. Hannon and G. T. Trammell, Phys. Rev. 169, 315 (1968).

${ }^{4}$ J. P. Hannon and G. T. Trammell, Phys. Rev. 186, 306 (1969).

${ }^{5}$ G. T. Trammell and J. P. Hannon, Phys. Rev. B 18, 165 (1978).

${ }^{6}$ J. P. Hannon and G. T. Trammell, Physica B 159, 161 (1989).

${ }^{7}$ J. P. Hannon and G. T. Trammell, in Resonant Anomalous X-Ray Scattering, edited by G. Materlik, C. J. Sparks, and K. Fisher (Elsevier, Amsterdam, 1994), p. 565.

${ }^{8}$ A. M. Afanas'ev and Yu. Kagan, Zh. Eksp. Teor. Fiz. 48, 327 (1965) [Sov. Phys. JETP 21, 215 (1965)].

${ }^{9}$ A. M. Afanas'ev and Yu. Kagan, Zh. Eksp. Teor. Fiz. 52, 191 (1967) [Sov. Phys. JETP 25, 124 (1967)].

${ }^{10}$ Yu. Kagan, A. M. Afanas'ev, and I. P. Perstnev, Zh. Eksp. Teor. Fiz. 54, 1530 (1968) [Sov. Phys. JETP 27, 819 (1968)].

${ }^{11}$ Yu. Kagan, A. M. Afanas'ev, and V. G. Kohn, J. Phys. C 12, 615 (1979).

${ }^{12}$ W. Sturhahn and E. Gerdau, Phys. Rev. B 49, 9285 (1994).

${ }^{13}$ B. Batterman and H. Cole, Rev. Mod. Phys. 36, 681 (1964).

${ }^{14} \mathrm{~W}$. Heitler, The Quantum Theory of Radiation, 3rd ed. (Oxford University Press, New York, 1957), p. 163.

${ }^{15}$ S. M. Harris, Phys. Rev. 124, 1178 (1961).

${ }^{16}$ G. R. Hoy, J. Phys. C 9, 8749 (1997).

${ }^{17}$ C. Kittel, Introduction to Solid State Physics, 3rd ed. (Wiley, New York, 1971)

${ }^{18}$ G. R. Hoy (unpublished).

${ }^{19}$ J. B. Hastings, D. P. Siddons, U. van Bürck, R. Hollatz, and U. Bergmann, Phys. Rev. Lett. 66, 770 (1991).

${ }^{20}$ U. van Bürck, D. P. Siddons, J. B. Hastings, U. Bergmann, and R. Hollatz, Phys. Rev. B 46, 6207 (1992).
${ }^{21}$ S. Kikuta, in Resonant Anomalous X-Ray Scattering (Ref. 7), p. 635 and references therein.

${ }^{22}$ U. Bergmann, J. B. Hastings, and D. P. Siddons, Phys. Rev. B 49, 1513 (1994).

${ }^{23}$ M. Seto, Y. Yoda, S. Kikuta, X. W. Zhang, and M. Ando, Phys. Rev. Lett. 74, 3828 (1995).

${ }^{24}$ W. Sturhahn, T. S. Toellner, E. E. Alp, X. Zhang, M. Ando, Y. Yoda, S. Kikuta, M. Seto, C. W. Kimball, and B. Dabrowski, Phys. Rev. Lett. 74, 3832 (1995).

${ }^{25}$ A. I. Chumakov, R. Rüffer, H. Grünsteudel, H. F. Grünsteudel, G. Grübel, J. Metge, O. Leupold, and H. A. Goodwin, Europhys. Lett. 30, 427 (1995).

${ }^{26}$ A. Q. R. Baron, A. I. Chumakov, R. Rüffer, H. Grünsteudel, H. F. Grünsteudel, and O. Leupold, Europhys. Lett. 34, 331 (1996).

${ }^{27}$ A. I. Chumakov, R. Rüffer, A. Q. R. Baron, H. Grünsteudel, and H. F. Grünsteudel, Phys. Rev. B 54, 9596 (1996).

${ }^{28}$ W. Sturhahn, K. W. Quast, T. S. Toellner, E. E. Alp, J. Metge, and E. Gerdau, Phys. Rev. B 53, 171 (1996).

${ }^{29}$ G. V. Smirnov and V. G. Kohn, Phys. Rev. B 52, 3356 (1995).

${ }^{30}$ X. Zhang, Y. Yoda, M. Seto, Yu. Maeda, M. Ando, and S. Kikuta, Jpn. J. Appl. Phys., Part 2 34, L330 (1995).

${ }^{31}$ A. Q. R. Baron, A. I. Chumakov, S. L. Ruby, J. Arthur, G. S. Brown, G. V. Smirnov, and U. van Bürck, Phys. Rev. B 51, 16384 (1995).

${ }^{32}$ V. A. Belyakov, Zh. Eksp. Teor. Fiz. 108, 741 (1995) [JETP 81, 405 (1995)].

${ }^{33} \mathrm{R}$. W. James, The Optical Principles of The Diffraction Of X-Rays (Ox Bow Press, Woodbridge, CT, 1982), p. 138.

${ }^{34}$ G. V. Smirnov, Hyperfine Interact. 123/124, 31 (1999).

${ }^{35}$ B. Sepiol, A. Meyer, G. Vogl, R. Rüffer, A. I. Chumakov, and A. Q. R. Baron, Phys. Rev. Lett. 76, 3220 (1996). 